\title{
Shared Viewpoint-Developing the Future of Kidney Care
}

\author{
Joseph Vassalotti, $M D^{7}$, Clarissa Jonas Diamantidis, $M D^{2}$, and David J. Cook, $M D^{3} \oplus$ \\ ${ }^{1}$ National Kidney Foundation, Icahn School of Medicine at Mount Sinai, New York City, USA; ${ }^{2}$ Duke University School of Medicine, Durham, USA; \\ ${ }^{3}$ OptumLabs at United Health Group, Minneapolis, USA.
}

$\mathrm{J}$ Gen Intern Med 37(4):947-8

DOI: $10.1007 / \mathrm{s} 11606-021-07014-\mathrm{x}$

(c) Society of General Internal Medicine 2021

$\mathrm{T}$ he events of the last year will inevitably change how healthcare is perceived and delivered. Similarly, the ongoing health challenges of diabetes and hypertension, and the rising incidence of chronic kidney disease (CKD), compel us to consider the path towards optimal kidney health for the US population.

In addition to the tragic and immediate loss of life, the pandemic resulted in dramatic decreases in care delivery to those with chronic diseases. Patients with kidney disease are particularly vulnerable to both COVID-19 and the loss of care that occurred during the pandemic. It remains to be seen how deficits in care in 2020 will affect those with chronic conditions. Despite the current pandemic, the majority of the CKD population, particularly early stages, continues to be managed in the primary care setting.

Thirty-seven million adults in the United States are affected by chronic kidney disease (CKD) - 1 in every 7 adults - and approximately $90 \%$ are unaware of their condition. This blind spot in our health system is unacceptable. Lack of awareness and underdiagnosis of kidney disease leaves patients, families, and the healthcare system blind to a huge population's future risk of cardiovascular events, reduced quality of life, potential kidney failure, and death.

Changing this reality will require principles and solutions that get upstream of this silent disease by improving CKD screening, better engaging patients, shifting incentives to early screening and diagnosis, and increasing access to proven therapies. To that end, the following recommended priorities chart a course for the future of kidney care.

1) Improving screening, diagnosis, and documentation of kidney disease.

Although kidney disease is the ninth leading cause of death in the United States, many individuals do not receive

Received May 2, 2021

Accepted June 25, 2021

Published online August 30, 2021 appropriate testing for $\mathrm{CKD}$, and thus are unable to receive a diagnosis or timely management of their condition.

Recommended priorities:

- Update the United States Preventive Services Task Force 2012 recommendation for CKD screening to reflect the current evidence supporting routine screening for highrisk asymptomatic adults.

- Use the "kidney profile" laboratory assessment, including assessment of both estimated glomerular filtration rate (eGFR) and urinary albumin-to-creatinine ratio (uACR) in routine care for CKD identification and risk stratification.

- Understand the recommendations for raceless eGFR reporting anticipated from the final report of the National Kidney Foundation-American Society of Nephrology Task Force which will recognize race as a social rather than a biological concept. Implementing uniform eGFR reporting is one element in the broader comprehensive quest for kidney health equity that will require a national approach developed in collaboration with kidney care stakeholders.

- Deploy risk-based heat map stratification tools using both eGFR and UACR to inform appropriate education, management, and specialty referral for individuals at the highest risk of adverse outcomes.

- Embed clinical decision support tools for CKD into electronic medical records to help identify CKD cases earlier in priority populations and drive improvements in the quality of care patients receive.

Advance education of primary care clinicians; beginning in medical school, continuing in residency, fellowship and on to advanced practice levels; about CKD risk factors, testing, detection and interventions that are graded and proportional to the eGFR and uACR risk stratification or heat map.

2) Better engaging patients and focusing on personcentered kidney care.

Challenges with low awareness of CKD in both patients and clinicians, complex terminologies and treatment strategies, and the general asymptomatic nature of most kidney diseases all make person-centered kidney care a critical ideology to improving kidney health.

Recommended priorities:

- Evaluate and adopt evidence-based in-home and mobile tools for kidney care, such as home-based testing for CKD or CKD risk factors using self-administered 
laboratory tests, kidney care via telehealth using phone or video-based technologies, mobile applications for selfmanagement of CKD, and intuitive risk assessment tools for patients with CKD and at risk for CKD.

- Examine patient acceptability of telehealth as a model for kidney care and potential barriers to expanding adoption.

- Disseminate clear and simple layperson-centered language in all clinician communications related to CKD self-management to enhance patient understanding and empowerment.

- Facilitate electronic sharing of patient CKD records without delay and improve the consistency of kidney care terminology and documentation in records.

- Promote home-based kidney replacement therapies such as home hemodialysis and peritoneal dialysis to optimize CKD self-management and patient choice that can be supported by primary care clinicians.

3) Moving the focus of CKD reimbursement upstream in the CKD continuum.

In 2018, Medicare expenditures alone for Americans living with kidney diseases exceeded 120 billion of which over 70 billion was allocated for the care of CKD without dialysis or kidney transplant. The additional total commercial health insurance expenditures for CKD are likely to be proportional to 70 billion. Improved CKD population health will limit cardiovascular hospitalization, reduce transitions to more advanced stages, and reduce ESKD. Collectively, these improved outcomes will reduce expenditures. Most patients with CKD will not progress to ESKD. The primary care clinician can play an important role to reduce cardiovascular risk throughout the spectrum of CKD, particularly in early-stage CKD.

Recommended priorities:

- Develop key performance indicators that further connect early-stage CKD detection with evidenced-based therapeutic interventions, and link performance to clinician reimbursement incentives.

- Shift federal initiatives and reimbursement strategies earlier in the CKD continuum, focusing on primary prevention of $\mathrm{CKD}$ and optimization of risk factors for CKD progression.

- Integrate new HEDIS measures, such as the Kidney Health Evaluation for Patients with Diabetes measure, into the Merit-based Incentive Payment System (MIPS) for Medicaid and Medicare quality measure programs and payment models to promote early screening and diagnosis.

4) Increasing access to evidence-based therapies for CKD patients.

Widespread implementation of both established and more recent advances in medications with proven benefit in individuals with $\mathrm{CKD}$ or $\mathrm{CKD}$ risk factors has proven challenging. Statins are evidence-based therapies to reduce the risk of cardiovascular events in CKD. Randomized trials have demonstrated safety of stain-based therapies in CKD, but this drug class remains underutilized in approximately a third of the eligible population according to US health services research. Moreover, several recent studies have shown the significant kidney and cardiovascular protective effect of a class of medications known as sodium glucose co-transport2 (SGLT2) inhibitors in individuals with kidney disease and type 2 diabetes. A scientific workshop with over 80 experts agreed SGLT2 inhibitors should be used accordingly. More recent randomized trials show that the SGLT2 inhibitors are kidney and cardioprotective for individuals with CKD and/or heart failure even in the absence of diabetes. However, the slow integration of evidence-based therapies into clinical care compounded by the cost of this class of medications has delayed their adoption into routine kidney care. The glucagon-like peptide-1 receptor antagonists (GLP-1 RAs) comprise another type 2 diabetes medication class that has shown cardio-protection and may also offer additional kidney protection.

Recommended priorities:

- Update existing clinical guidelines to include recent advances in evidence-based treatments for kidney diseases.

- Appropriately expand the use of evidence-based medications for CKD, such as SGLT2 inhibitors and GLP-1 RAs, and reduce out of pocket expenditures for the patient to facilitate health equity.

- Promote scientific research on alternative therapies for the advancement of kidney health in high-risk populations.

Recommending this set of priorities is an initial step toward the future of kidney care. A future where each person is aware of their kidney health status as well as their blood pressure, cholesterol, and metabolic health. Awareness of health and health risk is critical to empowering patients, families, and their communities to take educated steps toward protecting kidney health and detecting signs of kidney damage as early as possible to expand patient choice in mitigating progression.

Corresponding Author: David J. Cook, MD; OptumLabs at United Health Group, Minneapolis, USA (e-mail: dcook@uhg.com).

Publisher's Note: Springer Nature remains neutral with regard to jurisdictional claims in published maps and institutional affiliations. 